www.jmscr.igmpublication.org

Impact Factor (SJIF): 6.379

Index Copernicus Value: 79.54

ISSN (e)-2347-176x ISSN (p) 2455-0450

crossrefDOI: https://dx.doi.org/10.18535/jmscr/v6i10.10

Journal Of Medical Science And Clinical Research

\title{
Prospective follow up study of idiopathic dilated cardiomyopathy in 112 patients at a tertiary care Hospital
}

Authors

\section{Saikat Sau, Sourav Sau}

B.S.M.C. Bankura

\begin{abstract}
Introduction: Idiopathic dilated cardiomyopathy is a mostly progressive irriversible disease with high global burden. A large number of cardiac and systemic diseases can cause systolic impairment and left ventricular dilatation but in majority of patient around twenty identifiable cause is found. Improvement in medical as well as device related strategy adherence to medical management and proper follow up can improve the morbidity and mortality of population effected by this disease.

Materials: Prospective follow up study of Hundred and twelve consecutively recruited patient over three years period Framingham's criteria for heart failure and echocardiographic evidence of low ejection fraction LVEF $<45 \%$ were included in our study. Clinical and echocardiographic evaluation and done at six month interval for assessment of improvement after medical treatment.

Result: Out of total 112 patients 16 patients had died due to cardiac cause either worsening of heart failure, sudden cardiac death, new onset acute coronary syndrome or ventricular arrhythmia. Overall improvement in LVEF and NYHA class achieved in $68 \%$ of patients at two years follow-up. Those who achieved the goal are excluded from the study group. 54\% of patient achieved NYHA class-I.

\section{Conclusion}

i. $\quad$ A substantial number of patients shows $L V$ reverse remodelling.

ii. $\quad$ Short duration of symptoms and LV end diastolic dimension at presentation are important predictor of recovery.

iii. Isotonic exercise is always encouraged in stable heart failure patients.

iv. LV end diastolic dimension and NYHA functional class are important predictor of event free survival and clinical recovery.

Keywords: Idiopathic dilated cardiomyopathy. Heart failure, NYHA class, Medical treatment in heart failure.
\end{abstract}

\section{Introduction}

Idiopathic Dilated cardiomyopathy is a progressive usually irreversible Disease causing global systolic dysfunction and heart failure. A large number of cardiac and systemic diseases can cause systolic impairment and left ventricular dilatation but in majority of patients no identifiable cause is found, hence the term idiopathic is used. It affects more than 36 individual per one lakh population. IDCM Accounts for fifty thousand hospital admission and ten thousand deaths each year. During past two decades survival of IDCM is improved after major advances in pharmacological and device based therapy for heart failure. 
Real world implementation of standard heart failure guideline is challenging, compliance is lower than reported in clinical trials documenting survival benefit. As a result intermediates outcome of DCM in community remain largely unreserved. We therefore choose to examine the clinical treatment and prognosis of a sizeable cohot of unselected consecutively enrolled patient with DCM from a well define regional population evaluated over 3 yrs in a systemic fashioned by the same team. Treatment strategy includes evidence based pharmacological treatment and serial investigation for follow up.

\section{Materials \& Methods}

We have studied 112 patients in local population over 3 years period. The population was mainly tribal based 21 to 74 yrs age. Patient admitted in our Hospital \& Subsequently discharged and followed up at our OPD. Patient with previous history of IHD, H/O- revascularisation, echocardiographic evidence of regional wall motion abnormality ,ECG evidence of prior ACS or vascular heart disease are excluded from the study population. Clinical \& Echocardiographic criteria was used as sole diagnostic modality to diagnosis of heart failure patients. Patient with NYHA class 3 \& 4 were admitted for treatment. They all received the treatment as per protocol. Diagnosis of DCM was done by clinical signs of heart failure as per Framingham's criteria and or Echocardiographic features such as dilated globally hypokinetic LV with low ejection Fraction (LVEF <45\%) . Patient with NYHA Class IV were treated at ICCU as indicated medication and added inotrophic or Vasopressure medication when required. Discharged patients were followed up at OPD In regular interval with all medicines available at OPD in our Hospital. Serial Echocardiographic assessment with ECG, Blood, Biochemistry and functional class assessment done at 3 months interval and when required. All stable patients were discharged and follow up at Cardiology OPD.
They were readmitted when become symptomatic NYHA III/IV. During follow up study they were encouraged to do normal household work\& Isotonic exercise irrespective of LVEF status. All patients were advised to adhere on prescribe medicine and not to do isometric exercise such as weight lifting or pumping tube well. Ischemic etiology of DCM excluded by only non invasive means as cat-lab facility not available and most patient refuse to undergo invasive procedure.

\section{Result}

Patient recruitment and follow up are summarised. The study was done from January 2015 to December 2017 a total 112 patients follow up. All patients were diagnosed with IDC prior to inclusion. Clear cut diagnosis of IDC Included in our study. Two patient prior inflammatory myocardial disease as we diagnosed by CMR , one patient with non compaction as diagnosed by CMR are excluded from the study group.

Patient treatment during two years follow up with medicine:- TABLE-(2)

At 6 month follow up hundred and eight patient were re examined. Two patients did not attend the follow up, one patient had died due to heart failure, one patient undergone resynchronisation therapy at hospital in South India. At one year follow up one more patients had died at home due to sudden cardiac arrest, two patients died in our Hospital after admission due refractory heart failure and pneumonia respectively. One patient not attend two schedule follow up.

Prior to initiation of treatment of a large majority were severely symptomatic. During follow up there was a significant change of NYHA class (P $<.001)$. Most of the clinical benefit occur after initiation of treatment.

A substantial number of Patient shows increase in LVEF as detected by Echocardiography during first 6 month follow up . From baseline to 1st year follow up fifty two percent shows increase in LVEF by $7 \%$. Seven percent shows decrease in LVEF by $5 \%$ and rest of the patients shows insignificant improvement in follow up. During 


\section{JMSCR Vol||06||Issue||10||Page 64-68||October}

the eighteen month follow up fourteen patient shows complete recovery of LV function detected by LVEF $>50 \%$. These patients also shows normal hsCRP \& Nt proBNP initially which was increased. Duration of symptoms, ECG changes during the Symptom and LVEdD at presentation were important parameter with short term improvement after start of treatment. Only LVEdD and duration of symptom not the LVEF remains independent predictor of morality in multivariate model.

Changes in parameter after the first year follow up.

After a median eighteen month follow up eight patients were died. Two of them had unusual death not related to cardiac cause, one of them from accident another one from CVA. Only one patient undergone CRT.
Baseline predictor of LV ejection fraction after 18 month. At two years follow up out of 112 patients included in study sixteen patients had died due to cardiac cause, either worsening of HF, Sudden cardiac death, new onset acute coronary syndrome and ventricular arrhythmia. Overall improve LVEF AND NYHA class achieved in $68 \%$ of patient at two years follow up. Patient who achieved the goal were excluded from the study. Those not achieved the estimated parameter were extended to three years follow up. Another 12\% patients shows significant improvement in LVEF and NHYA class at 3 yrs follow up.

\section{Table 1}

Duration of study $3 \mathrm{yrs}$
Total patients population 112
Male- $60 \%(\mathrm{n}=68)$
Female- $40 \%(\mathrm{n}=44)$
Age - 19 to $76 \mathrm{yrs}$
Mean Ages $56 \mathrm{yrs}$

Table 2 Medicines used during initiation and followed up in patient populations.

\begin{tabular}{|l|c|c|c|c|}
\hline & Zero month & Six month & twelve month & Twenty four month \\
\hline Loop Diuretics & $100 \%$ & $100 \%$ & $93 \%$ & $86 \%$ \\
\hline ACE inhibitor/ARBs & $100 \%$ & $100 \%$ & $100 \%$ & $100 \%$ \\
\hline Digoxin & $56 \%$ & $24 \%$ & $18 \%$ & $14 \%$ \\
\hline Betablocker & $58 \%$ & $72 \%$ & $68 \%$ & $84 \%$ \\
\hline Inhibitor & $81 \%$ & $76 \%$ & $80 \%$ & $74 \%$ \\
\hline Vasodilators & $43 \%$ & $54 \%$ & $47 \%$ & $57 \%$ \\
\hline Nitrate & $12 \%$ & $08 \%$ & $08 \%$ & $07 \%$ \\
\hline Inotrophic agents & $14 \%$ & $08 \%$ & $04 \%$ & $0 \%$ \\
\hline
\end{tabular}

Table 3 Clinical symptoms and signs in patient population at diagnosis and followup

\begin{tabular}{|l|c|c|c|c|}
\hline & zero month & six month & twelve month & Twenty four month \\
\hline Dyspnoea & $100 \%$ & $34 \%$ & $32 \%$ & $12 \%$ \\
\hline Palpitation & $87 \%$ & $42 \%$ & $37 \%$ & $15 \%$ \\
\hline Chest pain & $13 \%$ & $11 \%$ & $14 \%$ & $07 \%$ \\
\hline Effort in tolerance & $96 \%$ & $58 \%$ & $36 \%$ & $24 \%$ \\
\hline Pedal edema & $48 \%$ & $09 \%$ & $05 \%$ & $02 \%$ \\
\hline Engorged JVP & $78 \%$ & $15 \%$ & $09 \%$ & $03 \%$ \\
\hline$S_{3}$ gallop & $69 \%$ & $03 \%$ & $04 \%$ & $02 \%$ \\
\hline Basal crepitation & $73 \%$ & $46 \%$ & $24 \%$ & $12 \%$ \\
\hline
\end{tabular}

\section{Discussion}

In our study mean improvement of LVEF was 15 $\%$ and average reduction in end diastolic diameter was $30 \%$. A substantial number of Patients shows LV reverse remodelling. Predictor of improvement in LV function was short duration of symptoms and LV end diastolic diameter at presentation. Possibly this variables are surrogated marker of acute at least partially reversible pathogenic process. As we did not proceed to cardiac Biopsy and MRI Definite Diagnosis was made in limited number of Patients only. We found that even patient with a probable definite etiology LVEF improved substantially. From this point it is concluded that though co pathogenic factors have important role, patient symptoms 
improve considerably irrespective of etiology. Clinical recovery and myocardial recovery has to be wined to denote the clinical freedom from feature heart failure and normalisation of LV structure and function. $30 \%$ of patients experienced myocardial function improvement during our three year medical management defined as LVEF More than 50\% with functional class NHYA $\leq$ II . Biochemical parameters such as it probably remained above the reference value in half of the remission patient suggestive of underline diseases remain though medical treatment continuing. Some patient experience SCD though their BNP levels were below the Average limit. Thus the normalise of systolic function in Idopathic Dilated cardiomyopathy does not necessarily signify the absence of pathology and hence forth cessation of medical treatment not suggested compare to ischemic heart failure where a significant portion of myocardial got irreversible damage in viability testing patient with IDC is not necessarily irreversible damaged. Therefore the pathogenesis of IDC might retain a potential for almost recovery given that drivers of the pathogenic process can be reversed it might be Spontaneous of removal of underlying process or due to optimal medical treatment.

It is assumed that the favourable prognosis observed in our patient population are due to strict adherence to current heart failure guideline, free supply of medicines, proper counselling of the patients and their relatives about the nature, sequel $\&$ prognosis of the disease process. Prognosis in heart failure in linked to LV size \& function. Remodelling is associated with adverse outcome. Significant population shows substantial increase in LVEF over the first year of follow up. Overall survival was fair and better than previously described. Population that shows moderate or no improvement remains same or only modest improvement in subsequent follow up. As the referral practice became more liberal more patient population could be recruited in the group and shows the positive benefit of treatment.
All patient in our population recruited in standard protocol of medical management as per guideline if not contraindicated.

The level dilated Cardiomyopathy cover a very heterogenous group of disease as far as both etiology and clinical menifestation concerned. Thus a very fastidious characteristic and reporting of the actual sample is required for the result to be reproducible and direct comparison with the historic cohort must be made with case.

\section{References}

1. Matsumura Y, Takata J, Kitaoka H, Kubo T, Baba Y, Hoshikawa E, Hamada T, Okawa M, Hitomi N, Sato K, Yamasaki N, Yabe T, Furuno T, Nishinaga M, Doi Y. Long-term prognosis of dilated cardiomyopathy revisited: an improvement in survival over the past 20 years. Circ J. 2006;70:376-383

2. Orio A.L. Pankuweit S., Arbustini E.,et al. (2013) Current state of knowledge o aetiology, diagnosis, management, and therapy of myocarditis: a position statement of the European Society of Cardiology Working Group on Myocardial and Pericardial Diseases. Eur Heart $\mathbf{J}$ 34:2636-2648, 2648a-d.

3. McMurray J.J.V., Adamopoulos S., Anker S.D., et al., for the ESC Committee for Practice (2012) ESC guidelines for the diagnosis and treatment of acute and chronic heart failure 2012 . Eur Heart J 33:1787-1847.

4. De Campos Lopes CB, Yamada AT, Araújo F, Pereira Barreto AC, Mansur AJ. Socioeconomic factors in the prognosis of heart failure in a Brazilian cohort. Int $\mathbf{J}$ Cardiol. 2006;113:181-7 Medline Yancy C.W.,

5. Jessup M., Bozkurt B., et al. (2013) 2013 ACCF/AHA guideline for the management of heart failure: a report of the American College of Cardiology Foundation/ American Heart Association Task Force 
on Practice Guidelines. J Am Coll Cardiol 62:e147-e239.

6. Stolfo D., Merlo M., Pinamonti B., et al. (2015) Early improvement of functional mitral regurgitation in patients with idiopathic dilated cardiomyopathy. Am J Cardiol 115:1137-1143.

7. Merlo M., Pyxaras S.A., Pinamonti B., et al.(2011) Prevalence and prognostic significance left ventricular reverse remodeling in dilated cardiomyopathy receiving tailored medical treatment. J Am Coll Cardiol 57:1468-1476.

8. Fatkin D., Yeoh T., Hayward C.S., et al (2011) Evaluation of left ventricular enlargement as a marker of early disease in familial dilated cardiomyopathy. Circ Cardiovasc Genet 4:342-348

9. Goldberger J.J.,Subačius H., Patel T., et al. (2014) Sudden cardiac death risk stratification in patients with nonischemic dilated cardiomyopathy. J Am Coll Cardiol 63:1879-1884

10. Merlo M, Pivetta A, Pinamonti B, Stolfo D, Zecchin M, Barbati G, et al. Long-term prognostic impact of therapeutic strategies in patients with idiopathic dilated cardiomyopathy: Changing mortality over the last 30 years.Eur J Heart Fail 2014; 16: 317-324.

11. JCS Joint Working Group. Guidelines for management of dilated cardiomyopathy and secondary cardiomyopathy (JCS2011) http://www.jcirc.or.jp (accessed July 31, 2014).

12. Takada T, Sakata Y, Miyata S, Takahashi J, Nochioka K, Miura M, et al; CH 2 Investigators. Impact of elevated heart rate on clinical outcomes in patients with heart failure with reduced and preserved ejection fraction. A report from the CHART-2 Study. Eur J Heart Fail 2014; 16: 309-316. 